\title{
Mid-infrared supercontinuum generation in suspended core tellurite microstructured optical fibers
}

\author{
M.Belal,* L.Xu, P.Horak, L.Shen, X.Feng, M.Ettabib, D.J.Richardson, \\ P.Petropoulos and J.H.V.Price \\ Optoelectronics Research Centre, University of Southampton, Southampton, SO17 1BJ, U.K. \\ *Corresponding author: mob@orc.soton.ac.uk
}

\begin{abstract}
We report the fabrication of a tellurite optical fiber with a suspended core design, formed on a $220 \mathrm{~nm}$ wide filament of glass. The fiber was pumped at two different wavelengths (1500 $\mathrm{nm}$ and $2400 \mathrm{~nm})$ using femtosecond pulses generated from an optical parametric oscillator (OPO) in order to produce mid-infrared supercontinuum (SC). We observed that SC spectra extending to $3 \mu \mathrm{m}$ were readily generated. To further optimize the design detailed numerical study was performed which revealed how the fiber structural characteristics dramatically influence the spectral broadening because of the changes in the dispersion profile and in turn, the interplay of nonlinear effects that give rise to SC generation. We found that an accurate control of the core shape can be employed to contain the generated SC spectra within well-defined spectral regions or to provide a broad extension of the continuum to beyond $4 \mu \mathrm{m}$.
\end{abstract}

Fiber-based supercontinuum (SC) sources have an attractive combination of high brightness and broad bandwidth, which makes them ideal sources for spectroscopy [1] and if the coherence is high, then also for metrology [2]. Supercontinuum generation (SCG) across the mid-infrared (mid-IR) has enabled the molecular fingerprinting of organic compounds leading to a host of cross-disciplinary applications in the fields of sensing [3], cosmetic product inspection [4], protein structure derivation [5] and detection of biological species [6,7]. To extend the wavelength range beyond the transparency window of silica, mid-IR SCG has often been based on soft glasses [8] such as chalcogenide [9], tellurite (Te) [10,11], lead silicate [12] and fluoride [13]. Microstructuring has been used to engineer the zero-dispersion wavelength (ZDW), which is typically in the mid-IR for soft-glasses, to match the wavelength of widely available near-IR pulsed lasers. However, because of the narrow temperature range of the glass transition in soft glasses, the fibers are challenging to fabricate when compared to the now established procedures used to fabricate silica microstructured fibers. For this reason, as well as for the rather low mechanical robustness of these glasses, the innovation in Te and chalcogenide fiber designs has been quite limited as compared to silica fibers [14-16].

In this paper, we present experimental and modelling results showing SCG in a novel Te fiber with an elongated core on a thin filament of glass as shown by the scanning electron microscope (SEM) image in Fig. 1. The preform was fabricated using the extrusion method $[17,18]$ and was then drawn into the final fiber using a conventional fiber drawing tower. The fiber has high birefringence for compatibility with planar waveguides, and the shape of the fiber core is controlled both by the shape of the extrusion die and by surface tension effects during fiber drawing. Hence, there is more design freedom than in $\mathrm{so}^{-}$ called wagon-wheel fibers $[10,19]$ where surface tension effects between the core and multiple supporting filaments govern the core shape.
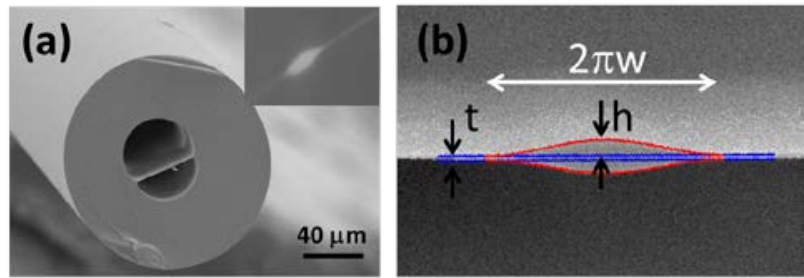

(c)

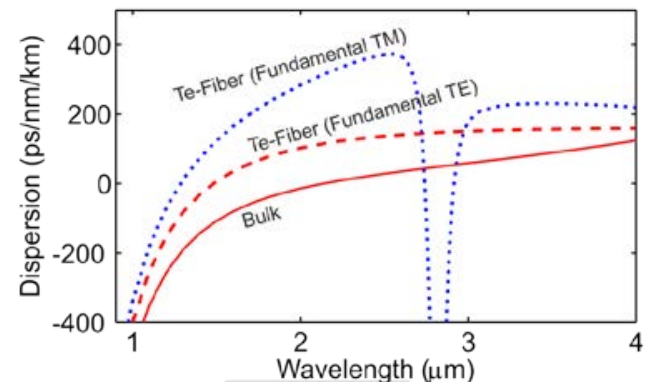

Fig. 1. (a) SEM image of the end facet of Te suspended core fiber with an inset depicting the core captured under an optical microscope. (b) Magnified SEM image of the guiding core and fitted outlines of the filament (straight blue line) and of the core (red) for numerical simulations. (c) Calculated dispersion profile of the fundamental TE and TM modes and that of bulk tellurite.

Alongside the experimental SCG results presented here, the simulations show the effect of the core shape on the SCG bandwidth. We simulated different cores, all with the same ZDW for the fundamental mode as the experimentally fabricated fiber, achieved by appropriately varying the major/minor axes. SCG simulations with identical initial pulses are then shown for the 
experimentally fabricated fiber and for the narrowest and widest of the core designs considered.

The tellurite glass composition used here was $70 \% \mathrm{TeO}_{2}$ $20 \% \mathrm{ZnO}-9 \% \mathrm{BaO}-1 \% \mathrm{BaCl}_{2} \quad$ (mol.\%). The glass was prepared in a dry atmosphere glove-box resulting in low levels of water retention of less than $0.2 \mathrm{ppm}$ [20]. This was crucial for reducing losses at wavelengths greater than $3 \mu \mathrm{m}$. The fiber loss is shown in Fig. 2(a). The 1.6 $\mathrm{dB} / \mathrm{m}$ loss at $1550 \mathrm{~nm}$ was measured using a $\mathrm{CW}$ laser and the cut back method. At longer wavelengths we estimated the loss from measurements on a bulk-glass sample (typically a good match with data on fabricated fibers). We have not calculated or measured the bend loss as it is not a major concern with the short fiber lengths considered here. Despite the dry processing there is a high loss at $3.4 \mu \mathrm{m}$, but a tellurite glass with a similar composition has enabled us recently to produce the improved loss curve shown in blue in Fig. 2(a) that should enable SCG out towards the $\sim 4.5 \mu \mathrm{m}$ multi-phonon edge. The dispersion profile of the fiber was calculated using a finite element method (FEM) based mode-solver. The dispersion of the glass was calculated based on the Sellmeier equation using published data for a related tellurite glass [21]. The core of the fabricated fiber could be fitted to a cosine function given by

$$
f(x)=h \frac{1}{2}\left(\cos \left(\frac{x}{w}\right)+1\right)
$$

where $h$ is the radius of the minor axis defined as the height of the core outline above the supporting filament and $w$ is the radius of the major axis characterized as the length of the core. The total thickness of the core is thus given by $2 h+t$, where $t$ is the thickness of the filament, and the elongated core is between $x=-\pi w$ and $+\pi w$. The excellent match of this profile to the fiber shape is shown by the line overlaid with the SEM image in Fig. 1(b). For the fabricated fiber the parameters were: $h=724 \mathrm{~nm}, w=$ $1.732 \mu \mathrm{m}, t=220 \mathrm{~nm}$ and the calculated dispersion is shown in Fig. 1(c). Following slab-waveguide nomenclature, we name the mode with E-field parallel to the filament as TE and the orthogonal mode as TM. The ZDW of the fundamental TE mode is $1480 \mathrm{~nm}$. The birefringence is so strong that the fundamental TM mode and first higher-order TE mode have almost degenerate mode-constants at a wavelength of $\sim 2.8 \mu \mathrm{m}$ resulting in a so-called avoided crossing, as evidenced by the dispersion anomaly at this wavelength shown in the graph. All of the SCG spectra in this paper are for the fundamental TE mode that produced the broadest $\mathrm{SC}$ in our simulations.

The fiber effective nonlinear coefficient of $\gamma=379 \mathrm{~W}^{-1} \mathrm{~km}^{1}$ [22] was measured using the nonlinear phase shift of a dual-frequency $\mathrm{CW}$ beat signal centred close to $1550 \mathrm{~nm}$. Figure $2(\mathrm{~b})$ shows this is in reasonable agreement with the calculated value from the FEM mode-solver, assuming an optical nonlinearity $n_{2}=2.5 \times 10^{-19} \mathrm{~m}^{2} / \mathrm{W}$ [23].
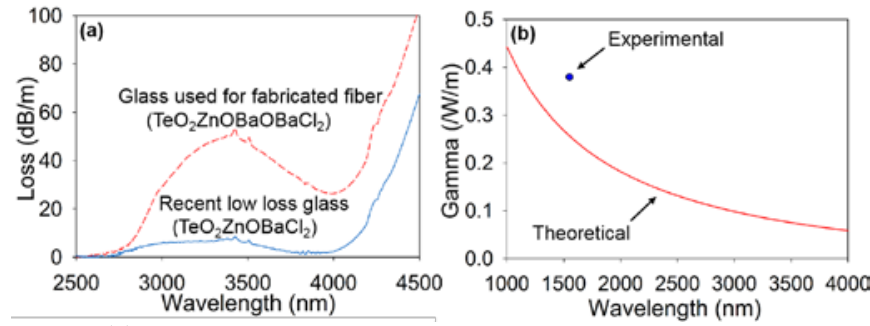

Fig. 2.(a) Measured loss of fabricated fiber and of our more recently made lower loss tellurite glass composition used in simulations for Fig's. 4,5; (b) Calculated non-linearity $(\gamma)$ values of the Te fiber as a function of wavelength and the experimentally obtained value at $1550 \mathrm{~nm}$.

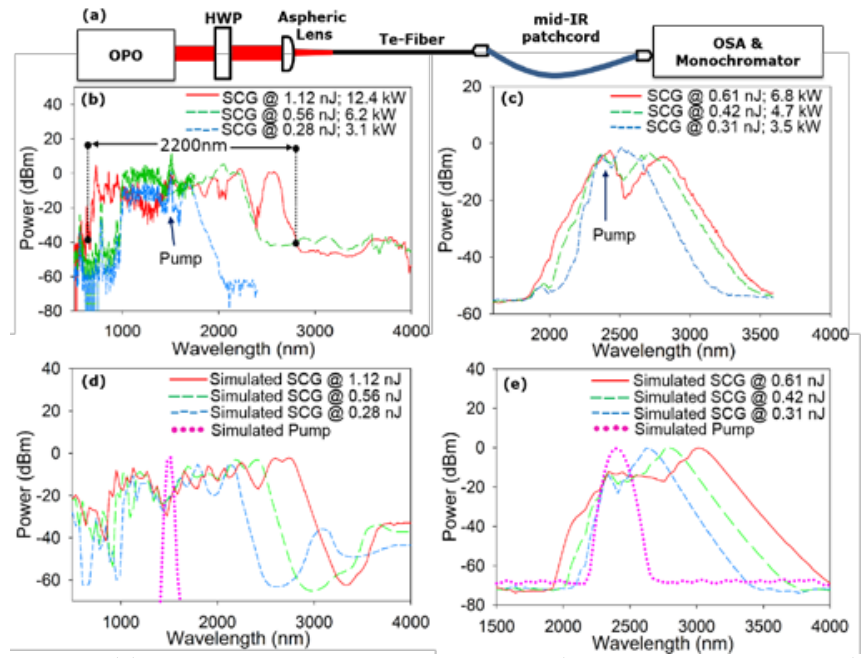

Fig. 3. (a) Schematic of the experiment (HWP-half wave-plate). Experimental output spectra vs. launched pulse energy when pumping at (b) $1500 \mathrm{~nm}$, (c) $2400 \mathrm{~nm}$. (d) and (e) simulated spectra corresponding to $(\mathrm{b})$ and $(\mathrm{c})$, respectively.

The experimental setup used for demonstrating SCG in the fiber is shown schematically in Fig. 3 (a). We launched $\sim 90$ fs linearly polarized pulses (time-bandwidth product $\sim 0.46$ ), from an in-house built optical parametric oscillator (OPO), similar to that reported in Ref. [24], using a halfwave-plate to couple into the TE mode that produced the broadest spectrum in a $15-\mathrm{cm}$ long fiber. The OPO output wavelength was $1500 \mathrm{~nm}$ with pulses at a repetition rate of $80 \mathrm{MHz}$ and average power of up to $400 \mathrm{~mW}$. The $\mathrm{n}_{\text {eff }}$ of the fundamental TE mode at $1550 \mathrm{~nm}$ is 1.98 so the Fresnel reflection is $10.8 \%$. With an aspheric lens ( $\mathrm{f}=$ $2.75 \mathrm{~mm}$ ) we coupled $\sim 22 \%$ of the power into the fiber. The achievable value considering mode-overlap with a circularly symmetric TEM00 Gaussian input with optimized beam diameter (1/e radius of the input intensity profile of $0.761 \mu \mathrm{m}$ ) would be $79.4 \%$ resulting in a possible coupling efficiency of $70.8 \%$ after the Fresnel loss, indicating improved mode-matching should be possible, but the coupled energy was still sufficient to achieve substantial spectral broadening. An indium fluoride patch cord was butt-coupled to the output to transport the light to a spectrometer. A Yokogawa OSA was used for wavelengths up to $2400 \mathrm{~nm}$ and a liquid-nitrogen cooled 
InSb detector attached to a monochromator (TMc300 from Bentham Ltd.) was used for longer wavelengths. Figure 3(b) shows the increase in the SC bandwidth with pump power. At the highest pump power of $400 \mathrm{~mW}$, corresponding to a launched pulse energy of $\sim 1 \mathrm{~nJ}$, a maximum SC bandwidth of $\sim 2200 \mathrm{~nm}$ was measured at a level $40 \mathrm{~dB}$ below the peak.

Next, to extend the SCG to longer wavelengths we launched the idler from the OPO, tuned to $2400 \mathrm{~nm}$ into a $3-\mathrm{cm}$ length of the fiber. The maximum OPO power was $290 \mathrm{~mW}$ at this wavelength and the coupling efficiency of $17 \%$ implied a maximum launched pulse energy of $\sim 0.5 \mathrm{~nJ}$. A pair of ZnSe lenses $(f=6 \mathrm{~mm})$ were used to couple the output into the indium fluoride patch cord connected to the monochromator. Figure 3(c) shows that the continuum then extended slightly beyond $3 \mu \mathrm{m}$.

As this experimental work used comparable pulse energies and durations to those in the work with a starshaped Te microstructured fiber (core size $2.5 \mu \mathrm{m}$ ) used in Ref. [19], which resulted in a continuum extending to $4 \mu \mathrm{m}$, we investigated the detailed influence of the pump wavelength in relation to the fiber ZDW and how the change in fiber shape influenced the continuum using numerical simulations. The simulations used the generalized nonlinear Schrödinger equation solved using a split-step Fourier method [25]. The equation contained the full dispersion profile of the fiber (Fig. 1), measured Raman response function for a similar glass [26] and the full loss profile of the fabricated fiber shown in Fig. 2(a). The nonlinearity was matched to the calculated value (Fig. 2(b)) at the pump wavelength and included the dominant $1 / \lambda$ wavelength dependent decrease at longer wavelengths, but, following common practice, it did not include the less significant $1 / \mathrm{A}_{\text {eff }}$ decrease with mode area. Figures $3(\mathrm{~d}, \mathrm{e})$ show the results using pulse parameters corresponding to those used experimentally in Figs. 3 $(b, c)$, confirming reasonable agreement between the simulations experiment. For both $1500 \mathrm{~nm}$ and $2400 \mathrm{~nm}$ pump wavelengths the majority of the broadening occurred rapidly due to soliton fission [25] in the first 3$5 \mathrm{~mm}$ of propagation along the fiber, followed by slower broadening due to soliton self-frequency shifting (SSFS) as the pulse propagated further.

In Ref. [19] the fiber ZDW was at $1380 \mathrm{~nm}$, so that the $1550 \mathrm{~nm}$ pump was $170 \mathrm{~nm}$ into the anomalous dispersion region. Simulations applying this offset to our fiber in Fig. 4 with $90 \mathrm{fs}, 1 \mathrm{~nJ}, 1650 \mathrm{~nm}$ seed pulses extended the continuum beyond $3 \mu \mathrm{m}$. In Fig. 4(a) the loss of the fabricated fiber has bee napplied, and this attenuates the long-wavelength edge so the broadest spectrum was achieved after $\sim 3 \mathrm{~cm}$ of propagation. This is consistent with our experimental observations that a $3 \mathrm{~cm}$ fiber length was approximately the optimum with the $2400 \mathrm{~nm}$ pump pulses. In Fig. 4(b) the results show that even with the lower loss of our more recently investigated tellurite glass the broadening slows rapidly at $\sim 3.5 \mu \mathrm{m}$ so additional broadening was only $\sim 700 \mathrm{~nm}$ compared to $1500 \mathrm{~nm}$ pumping. We expected that the monotonically increasing fiber dispersion coupled with the reduced nonlinearity at long wavelengths (see Fig. 2(b)) were the primary reasons.
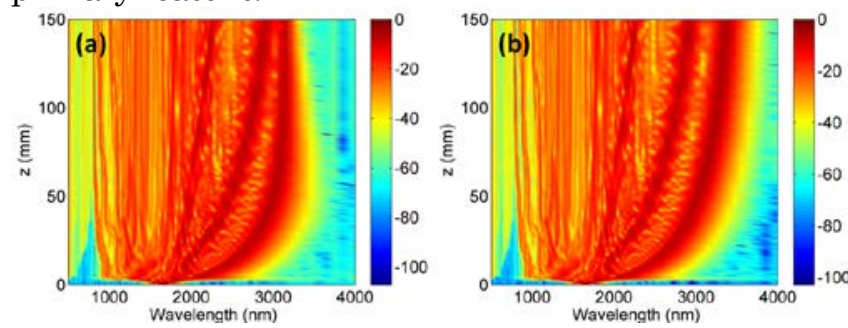

Fig. 4. Evolution of the supercontinuum spectrum (in $\mathrm{dB}$, normalized to the peak spectral density) vs fiber length pumping at $1650 \mathrm{~nm}$ and: (a) Fabricated fiber loss; (b) For our more recently made tellurite glass with lower loss (see Fig. 2(a)).

To investigate if a different, e.g. flatter, dispersion profile would enable further broadening and if that might be achieved by using the design freedom inherent in this fiber geometry of permitting core shape variation, we considered fiber designs in which the core was either more elongated or more circular than was the case for the experimentally fabricated fiber. We parameterized the search using the same cosinusoidal core shape that was found to be a good fit to the fiber in Fig. 1 and then used the full FEM based mode solver and a powerful computer to scan the parameter $w$ by multiplying it by a factor $q_{W}$ in the range 0.6 to 2 and then for each value of $q_{w}$ find a suitable multiplier $q_{h}$ to apply to the $h$ parameter that, for a constant value of $t$, kept the ZDW fixed at $1480 \mathrm{~nm}$ i.e. finding the appropriate transformation $(w, h) \rightarrow\left(w q_{w}, h q_{h}\right)$.

The predicted dispersion profiles for the extreme cases investigated, $q_{w}=0.6\left(q_{h}=1.3516\right)$ and $q_{w}=2\left(q_{h}=\right.$ 0.8074), are shown in Fig. 5(a) together with the original case $q_{W}=1\left(q_{h}=1\right)$; the corresponding fiber core shapes and mode functions at the pump wavelength are shown in Fig. 5(e,f). The more the design moved towards a circular shape (smaller $q_{w}$ ) the higher was the peak $\mathrm{D}$ value. The effective mode areas at $1500 \mathrm{~nm}$ wavelength for $q_{W}=0.6$, 1 , and 2 were computed as $3.73,3.93$, and $4.74 \mu \mathrm{m}^{2}$, respectively. The more circular cores had smaller mode areas compared to the most elongated ones. $\underline{\mathrm{SCG}}$ simulations for different values of $q_{w}$, and including the lower glass loss from Fig. 2(a) are shown in Fig. 5(b,c,d) as a new fiber could be fabricated from that newer glass composition. A pump wavelength of $1650 \mathrm{~nm}$ was again used with pulse energy of $1 \mathrm{~nJ}$ and duration of $90 \mathrm{fs}$. One might have expected that the reduced nonlinearity of the more elongated cores might limit the SC bandwidth. However, on the long wavelength edge, the SC is dominated by the SSFS of the fundamental solitons ejected by soliton fission [25]. Thus a lower dispersion peak produces a broader continuum output and to confirm this we compare Figs. 4 and 5(c, d), which show the evolution of the SCG along the fiber lengths for the three designs considered above. Again we found that the spectrum broadens rapidly until soliton fission occurs, 
then for the fiber with $q_{w}=0.6$ the SSFS comes to a stop at a wavelength of $\sim 3.5 \mu \mathrm{m}$ due to the rapidly increasing dispersion. The most elongated fiber core $\left(q_{w}=2\right)$ evolves similarly to the previous case until soliton fission occurs but then the SSFS continues to extend the spectrum to $\sim 4$ $\mu \mathrm{m}$. The flatter dispersion profile with smaller maximum $\mathrm{D}$ value has allowed substantial additional broadening.
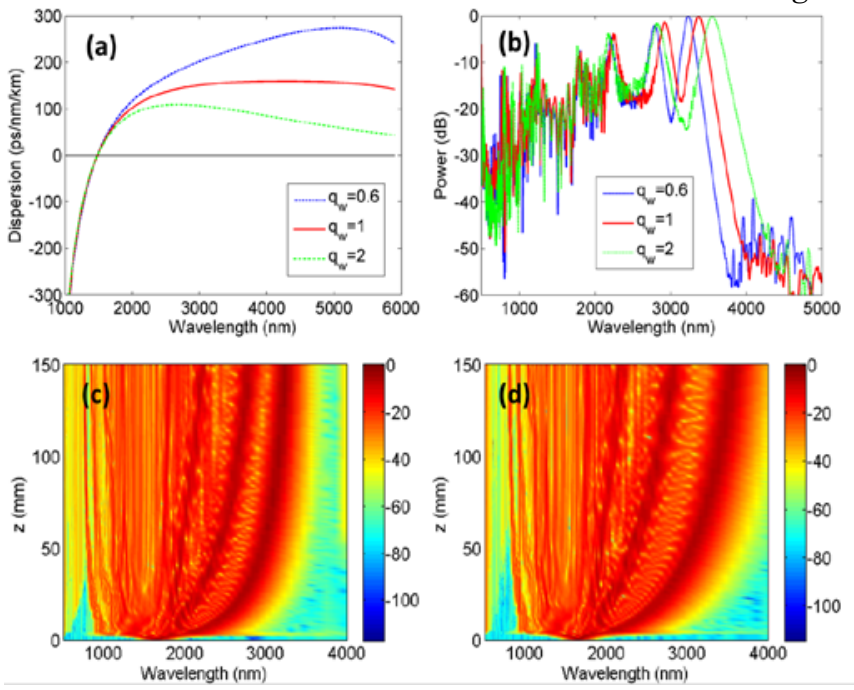

\section{(e)}

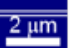

(f) $2 \mu \mathrm{m}$

Fig. 5. (a) Dispersion of fibers with different core shapes but same ZDW. (b) Simulated output spectra with $1650 \mathrm{~nm}, 400 \mathrm{~mW}$ average power seed $(80 \mathrm{MHz}$ repetition rate), (c), (d) Supercontinuum for $q_{w}=0.6$ and 2, respectively (in $\mathrm{dB}$, normalized). (For $q_{W}=1$ see Fig. 4.) (e), (f) Simulated modes for $q_{W}$ $=0.6$ and 2 at the pump wavelength.

In conclusion, we have fabricated a new suspended core Te fiber and have experimentally demonstrated SCG with fs pump pulses and extending to a maximum wavelength of $\sim 3 \mu \mathrm{m}$. We have illustrated by numerical simulations that making the fiber core shape more elongated while maintaining the same ZDW provides additional broadening to $\sim 4 \mu \mathrm{m}$ and provides higher form birefringence. In contrast, changing the core shape towards a circular profile led to the SCG being confined below $3 \mu \mathrm{m}$. This is the first investigation of a new Te fiber design with a core formed on a glass filament and provides insight into the trade-offs it enables for mid-IR generation from near-IR pump pulses.

This work was supported by the European Communities Seventh Frame-work Programme FP7/2007-2013 under Grant 288304 (STREP CLARITY) and by EPSRC (grants EP/I02798X/1, EP/H049371/1). We also thank Dr. Anna Peacock and Dr Trevor Newson for their assistance.

\section{References}

1. J.M.Langridge, T.Laurila, R.S.Watt, R.L.Jones, C.F.Kaminski and J.Hult, "Cavity enhanced absorption spectroscopy of multiple trace gas species using a supercontinuum radiation source", Opt. Express, 16(14), 10178 (2008)

2. A.Schliesser, N.Picqué and T.W.Hänsch "Mid-infrared frequency combs", Nature Photonics, 6, 440 (2012)

3. M.G.Allen, "Diode laser absorption sensors for gas" dynamic and combustion flows", Meas. Sci. Technol. 9, 545-562 (1998)

4. S.C.Kuo, S.K.Chuang, H.Y.Lin, and L.H.Wang, "Study of the aerosol fragrances of eugenol derivatives in Cananga odorata using diffuse reflectance infrared Fourier transform spectroscopy and gas chromatography." Analytica Chimica Acta, 653 (1), 91-96; (2009)

5. P.Rolfe, "In vivo near-infrared spectroscopy", Annu. Rev. Biomed. Eng. 02, 715-754 (2000).

6. B.Guo, Y.Wang, C.Peng, H.L.Zhang, G.P.Luo, H.Q.Le, C.Gmachl, D.L.Sivco, M.L.Peabody, and A.Y.Cho, "Laserbased mid-infrared reflectance imaging of biological tissues," Opt. Express 12, 208-219 (2004).

7. A.B.Seddon, "A prospective for new mid-infrared medical endoscopy using chalcogenide glasses", Int. J. Appl. Glass Sci. 2, 177-191 (2011)

8. J.H.V.Price, T.M.Monro, H.Ebendorff-Heidepreim, F.Poletti, P.Horak, V.Finazzi, Y.Y.Leong, P.Petropolis, J.C.Flanagan, G.Brambilla, X.Feng, and D.J.Richardson, "Mid-IR supercontinuum generation from nonsilica microstructured optical fibers," IEEE J. Sel. Top. Quantum. Electron.13, 738-750 (2007)

9. C.Rosenberg Petersen, U.Møller, I.Kubat, B.Zhou, S.Dupont, J.Ramsay, T.Benson, S.Sujecki, N.A.Moneim, Z.Tang, D.Furniss, A.Seddon and O.Bang, "Mid-infrared supercontinuum covering the 1.4-13.3 $\mu \mathrm{m}$ molecular fingerprint region using ultra-high NA chalcogenide step-index fibre," Nat. Photon. 8, 830 (2014)

10. I.Savelii, O.Mouawad, J.Fatome, B.Kibler, F.Désévédavy, G.Gadret, J-C Jules, P-Y Bony, H.Kawashima, W.Gao, T.Kohoutek, T.Suzuki, Y.Ohishi and F.Smektala, "Mid-infrared 2000-nm bandwidth supercontinuum generation in suspended-core microstructured Sulfide and Tellurite optical fibers", Opt. Express 16, 7161- 7167 (2008)

11. R. Thapa, D. Rhonehouse, D.Nguyen, K.Wiersma, C.Smith, J.Zong, A.C.Pirson, "Mid-IR supercontinuum generation in ultra-low loss, dispersion-zero shifted tellurite glass fiber with extended coverage beyond 4.5 um”, Proc. of SPIE 8898, 889808-1 (2013) 
12. F.G.Omenetto, N.A.Wolchover, M.R.Wehner, M.Ross, A.Efimov, A.J.Taylor, V.V.R.K.Kumar, A.K.George, J.C.Knight, N.Y.Joly, and P.St.J.Russell, "Spectrally smooth supercontinuum from $350 \mathrm{~nm}$ to $3 \mu \mathrm{m}$ in subcentimeter lengths of soft-glass photonic crystal fibers," Opt. Express 14, 4928-4934 (2006)

13. C.Xia, Z.Xu, M.N.Islam, F.L.Terry Jr., M.J.Freeman, A.Zakel, and J.Mauricio, "10.5 W Time-Averaged Power Mid-IR Supercontinuum Generation extending Beyond 4 $\mu \mathrm{m}$ With Direct Pulse Pattern Modulation”, Journal of Selected Topics in Quantum Electronics, 15(2), 422 (2009) 14. A.Bétourné, G.Bouwmans, Y.Quiquempois, M.Perrin, and M.Douay, "Improvements of solid-core photonic bandgap fibers by means of interstitial air holes", Opt. Lett., 32(12), 1719 (2007)

15. D.G.Ouzounov, F.R.Ahmad, D.Müller, N.Venkataraman, M.T.Gallagher, M.G.Thomas, J.Silcox,

K.W.Koch, A.L.Gaeta, "Generation of Megawatt Optical Solitons in Hollow-Core Photonic Band-Gap Fibers", Science, 3011702 (2003)

16. F.Poletti, N.V.Wheeler, M.N.Petrovich, N.Baddela, E.N.Fokoua, J.R.Hayes, D.R.Gray, Z.Li, R.Slavík, D.J.Richardson "Towards high-capacity fiber-optic communications at the speed of light in vacuum", Nature Photonics, 7, 279 (2013)

17. X.Feng, T.M.Monro, V.Finazzi, R.C.Moore, K.Frampton, P.Petropoulos, and D.J.Richardson, "Extruded singlemode, High-nonlinearity, tellurite glass holey fiber," Electron. Lett. 41(15), 835-837 (2005)

18. Z.Lian, P.Horak, X.Feng, L.Xiao, K.Frampton, N.White, J.A.Tucknott, H.Rutt, D.N.Payne, W.Stewart and W.H.Loh, "Nanomechanical optical fiber", Opt. Express, 20(28), 29386-29394 (2012)

19. P.Domachuk, N.A.Wolchover, M.Cronin-Golomb, A.Wang, A.K.George, C.M.B.Cordeiro, J.C.Knight, F.G.Omenetto, "Over $4000 \mathrm{~nm}$ Bandwidth of Mid-IR Supercontinuum Generation in sub-centimeter Segments of Highly Nonlinear Tellurite PCFs", Opt. Express 16, 7161- 7167 (2008)

20. Xian Feng, Jindan Shi, Martha Segura, Nicolas M.White, Pradeesh Kannan, Wei H.Loh, Laurent Calvez, Xianghua Zhang, and Laurent Brilland, "Halo-tellurite

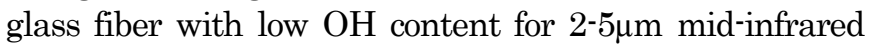
nonlinear applications, Opt. Express, 21(16), 1894918954 (2013)

21. G.Ghosh, "Sellmeier coefficients and chromatic dispersions for some tellurite glasses", J. Am. Ceram. Soc., 78(10), 2828-2830 (1995)

22. A.Boskovic, L.Gruner-Nielsen, O.A.Levring, S.V.Chernikov, and J.R.Taylor, "Direct continuous-wave measurement of $\mathrm{n}_{2}$ in various types of telecommunication fiber at 1.55 $\mu \mathrm{m}$," Opt. Lett., 21(24), 1966-1968 (1996).

23. L.L.Chase, E.W.V.Stryland and M.J.Weber, Ed., "Nonlinear optical properties," in Handbook of Laser Science and Technology Supplement 2: Optical Materials. Boca Raton, FL: CRC Press.
24. L.Xu, J.Feehan, L.Shen, A.Peacock, D.Shepherd, D.Richardson, and J.V.Price, "Yb-fiber amplifier pumped idler-resonant PPLN optical parametric oscillator producing 90 femtosecond pulses with high beam quality," Appl Phys B 117, 987-993 (2014).

25. J.M.Dudley, G.Genty, and S.Coen, "Supercontinuum generation in photonic crystal fiber," Rev. Mod. Phys., 78 (4), 1135-1184 (2006)

26. J. Shi, X. Feng., P. Horak, K. Chen, P. S. Teh, S. Alam, W. Loh, D. Richardson and M. Ibsen, "1.06 um Picosecond Pulsed, Normal Dispersion Pumping for Generating Efficient Broadband Infrared Supercontinuum in MeterLength Single-Mode Tellurite Holey Fiber With High Raman Gain Coefficient", J Lightwave Technol, 29(22), 3461-3469 (2011) 